\title{
An experimental study of the transient regime to fluidized chimney in a gran- ular medium
}

\author{
Pierre Philippe ${ }^{1, \star}$, Sarah Mena ${ }^{2}$, Florian Brunier-Coulin ${ }^{1}$, and Jennifer Curtis ${ }^{2,3}$ \\ ${ }^{1}$ IRSTEA, UR RECOVER, 3275 route de Cézanne, 13182 Aix-en-Provence, France. \\ ${ }^{2}$ Department of Chemical Engineering, University of Florida, Gainesville, FL 32611, Florida, US \\ ${ }^{3}$ College of Engineering, Department of Chemical Engineering, Kemper Hall, University of California, Davis, CA 95616, California, \\ US
}

\begin{abstract}
Localized fluidization within a granular packing along an almost cylindrical chimney is observed when an upward fluid-flow, injected through a small port diameter, exceeds a critical flow-rate. Once this threshold reached, a fluidized area is first initiated in the close vicinity of the injection hole before gradually growing upward to the top surface of the granular layer. In this work, we present an experimental investigation specifically dedicated to the kinetics of chimney fluidization in an immersed granular bed. Two different transient regimes are identified depending on wether the expansion of the fluidized area is rather fast and regular, reaching the final chimney state typically in less than 10 seconds, or, on the contrary, slow and very progressively accelerated, giving rise to transient duration up to 1 hour or even more. Some systematic investigations allow to propose several empirical scaling relations for the kinetics of chimney fluidization in the fast regular regime.
\end{abstract}

\section{Introduction}

Many industrial processes rely on particle bed fluidization, induced in most cases by a uniform fluid flow [1]. Much less often, the fluid injection can be restricted to a small area compared with the dimensions of the granular bed. Very little attention has been paid to this specific situation which generates only a partial fluidization of the system, through a vertical fluidized chimney, and defines lateral boundaries between fluidized and static zones. However, localized fluidization is relevant in some industrial applications, as spouted beds, but also in the particular context of embankment dam safety where such local structures, observed at the downstream foot of a levee and most commonly referred to as "sandboil", may indicate the presence, at an earlier stage, of an erosion process called backward erosion, listed as one of the four generic mechanisms of internal erosion [2]. Few experimental investigations have been conducted on steady-state localized fluidization, except the previous studies by Zoueshtiagh et al. [3] and by Philippe et al. [4], and even less regarding the transient upward expansion of the fluidized zone from an original cavity to a complete chimney [4].

Towards this end and for the purpose of expanding our previous preliminary study [4], an experimental study was carried out to investigate specifically this transient behaviour at the early stages of local fluidization. Our experimental procedure relies on the combination of Refractive Index Matching and Planar Laser Induced Fluorescence

\footnotetext{
^e-mail: pierre.philippe@irstea.fr
}

[4] allowing a direct visualization trough the granular material made of glass spheres immersed in an oil mixture. In addition to the flow rate at injection, the other parameters under study were the particle diameter (from $3 \mathrm{~mm}$ to $10 \mathrm{~mm}$ ), the injection diameter (from $10 \mathrm{~mm}$ to $40 \mathrm{~mm}$ ), and the initial granular bed height $\left(H_{0}=80 \mathrm{~mm}\right.$ or $\left.125 \mathrm{~mm}\right)$. A systematic analysis of the fluidized area expansion with time was then undertaken, based on the measurement of the total duration of the transient phase which allows distinguishing between a slow and very progressively accelerated transient regime and a faster and regular one. In this later regime, a more systematic investigation was carried out to study the kinetics of fluidized area expansion, including the dependencies with the different control parameters, and to propose some empirical scaling relations.

\section{Experiments}

The experimental investigation rely on the same optical techniques than used in our previous study [4]. These include Planar Laser Induced Fluorescence (PLIF) together with Refractive Index matching (RIM) [5]. RIM technique is based on the use of a solid phase and a liquid phase sharing the same refractive index and consequently giving rise to a translucent medium. After addition of a very small amount of fluorescent dye in the liquid, PLIF consists in illuminating the particulate system with a planar laser at a wavelength inside the fluorescence spectrum of the dye. Then an optical high-pass filter allows to recover a picture 


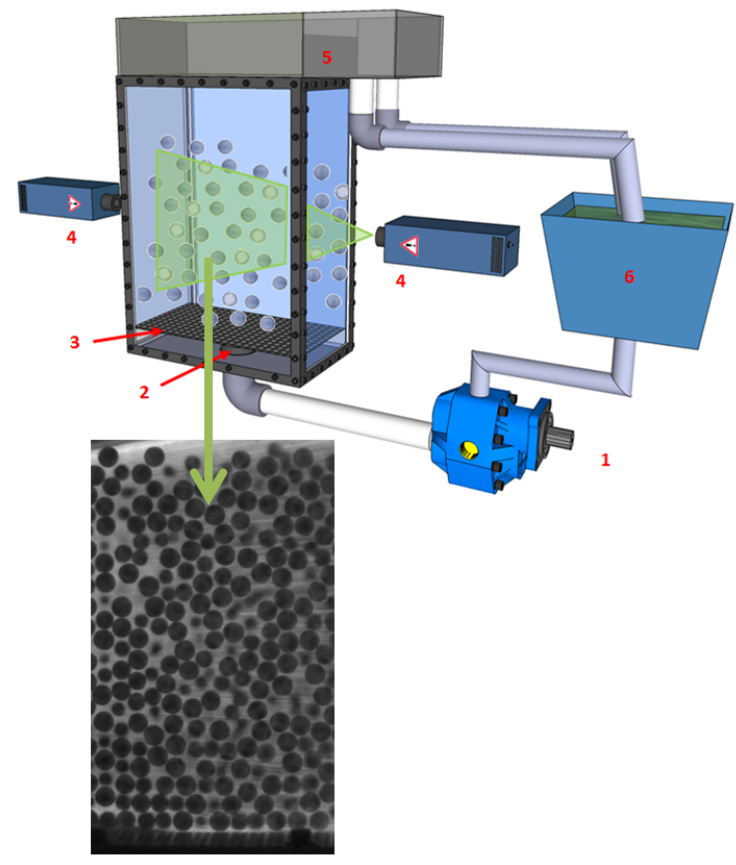

Figure 1. Experimental device (1: Gear pump; 2: Injection port; 3: Metallic mesh plate; 4. Planar lasers; 5: Over-flow set-up; 6: Reservoir) and typical picture of the granular bed obtained by coupling RIM and PLIF techniques.

where the microstructure is directly observed as illustrated in Figure 1.

In practice here, the solid phase is made of borosilicate glass beads (spherical shape) of density $\rho_{s}=2230 \mathrm{~kg} \cdot \mathrm{m}^{-3}$ and with particle diameter $d=3,5,7$ or $10 \mathrm{~mm}$. The liquid phase is a mixture with $90 \%$ (in mass) of light mineral oil and $10 \%$ of immersion oil. The liquid density is $\rho_{f}=840 \mathrm{~kg} \cdot \mathrm{m}^{-3}$ and the dynamical viscosity is approximately equal to $(28 \pm 2) \times 10^{-3} \mathrm{~kg} \cdot \mathrm{m}^{-1} \cdot \mathrm{s}^{-1}$ in the range of temperature experienced during this study. The refractive index of the liquid, which also approximately the one of the beads, was measured equal to 1.472 at $23^{\circ} \mathrm{C}$.

During an experiment, images similar the one in Figure 1 are recorded by a camera positioned perpendicularly to the laser plane. Two different cameras have been used with frame rate varying from 1 to $125 \mathrm{fps}$. A sketch of the experimental set-up is shown in Figure 1. It consists in a rectangular box (width $=10 \mathrm{~cm}$, height $=30 \mathrm{~cm}$, length $=20 \mathrm{~cm}$ ) filled by the beads sample on top of a horizontal metallic $2 \mathrm{~mm}$ mesh plate and over an injection port with changeable diameter $D=10 \mathrm{~mm}, 20 \mathrm{~mm}, 30 \mathrm{~mm}$, or $40 \mathrm{~mm}$. The liquid is supplied at a slow rate by a gear pump from a reservoir to saturate the granular medium in the cell and till the over-flow condition at the top of the device is reached. Next the pump is stopped to prepare the initial bed state. For this purpose, a metallic rod is manually and systematically stirred around within the granular bed both to ensure a good reproducibility and to eliminate any trapped bubbles. The global solid volume fraction obtained with this preparation method is approximately $\Phi=0.61 \pm 0.01$ for all bead diameters as estimated
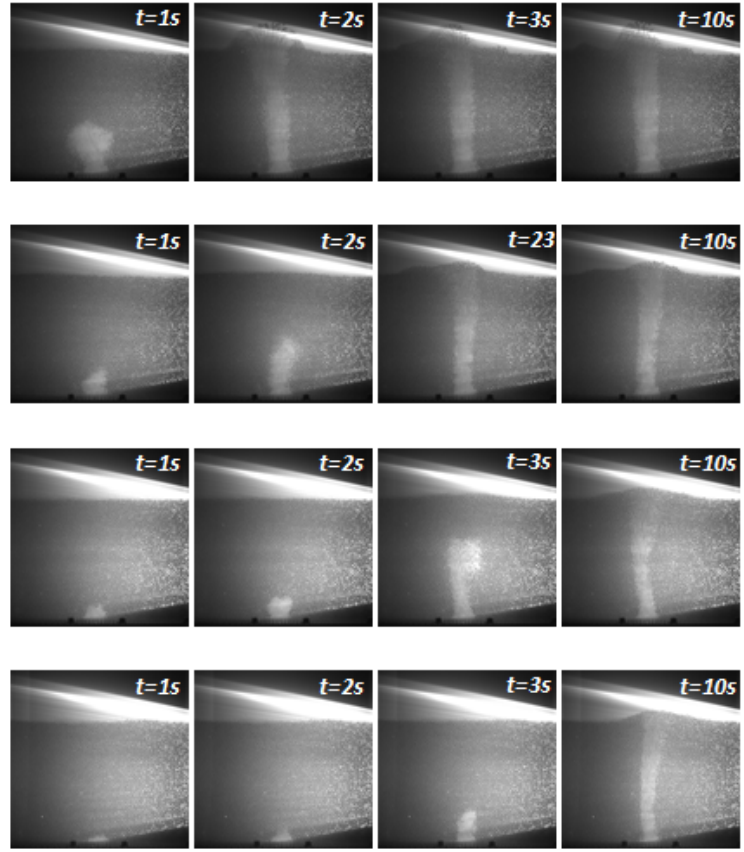

Figure 2. Sequences of transient fluidized area expansion ( $\left.d=3 \mathrm{~mm}, D=20 \mathrm{~mm}, H_{0}=125 \mathrm{~mm}\right)$ at successive time steps $t=1 s, t=2 s, t=5 s$, and $t=10 \mathrm{~s}$, with, from top to bottom, injection flow rates $Q$ and transient duration $T_{0}$ equal to $Q=117.5 \mathrm{~cm}^{3} \cdot \mathrm{s}^{-1}$ and $T_{0}=1.80 \mathrm{~s}, Q=68.1 \mathrm{~cm}^{3} \cdot \mathrm{s}^{-1}$ and $T_{0}=2.72 \mathrm{~s}, Q=50.4 \mathrm{~cm}^{3} \cdot \mathrm{s}^{-1}$ and $T_{0}=4.65 \mathrm{~s}, Q=44.6 \mathrm{~cm}^{3} \cdot \mathrm{s}^{-1}$ and $T_{0}=8.30 \mathrm{~s}$.

from the measurement of the initial bed's height $H_{0}$ which, in the present study, is chosen equal to either $H_{0}=80 \mathrm{~mm}$ or $H_{0}=125 \mathrm{~mm}$.

Starting from this reproducible initial sample, each experiment consists in imposing with the gear pump a prescribed constant flow-rate $Q$. The time needed for the pump to reach the selected flow-rate is small enough to be negligible compared to the transient time for fluidized chimney development. The later time, noted $T_{0}$, is highly sensitive to the flow-rate $Q$ as discussed in more details in the following section.

\section{Results}

\subsection{Transient regimes of chimney fluidization}

As shown in the pictures of Figure 2, the duration of the transient fluidized area expansion increases more and more markedly as the injection flow rate is reduced. For transient time typically less than 10 to 20 seconds, this expansion is rather regular with a rate of expansion which increases by a factor no more than 10 from the beginning of the process to the final chimney steady-state. In this regime, the relationship between $T_{0}$ and $Q$ is compatible with a power law divergence as shown in Figure 3:

$$
T_{0} \propto\left(\frac{Q}{Q_{c}}-1\right)^{-n}
$$

where $Q_{c}$ appears as a critical flow rate while the exponent $n$ remains between 1 and 0.5 . 


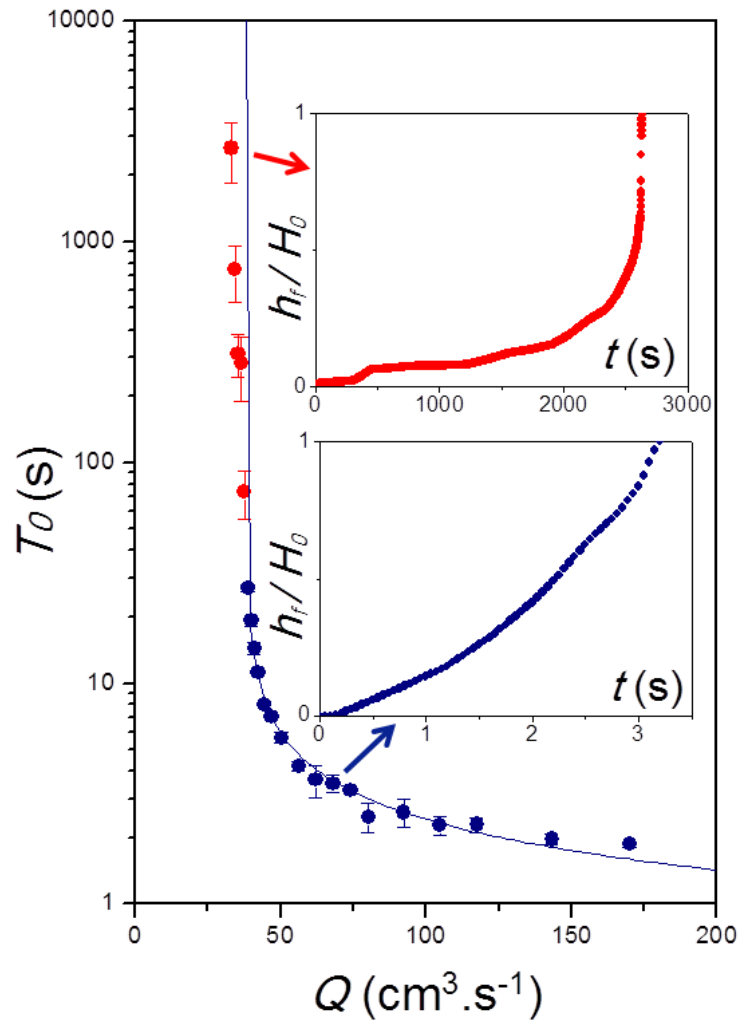

Figure 3. Transient duration $T_{0}$ versus injection flow rate $Q$ for $d=3 \mathrm{~mm}, D=30 \mathrm{~mm}$, and $H_{0}=125 \mathrm{~mm}$. The blue symbols account for the regular regime where a power law divergence is observed (Eq. 3.1 represented by the blue solid line) while the red symbols correspond to the ultra-slow regime as discussed in the text. Insets: typical time evolution of the fluidized height $h_{f}$ in the ultra-slow regime (top and red symbols) and in the regular regime (bottom and blue symbols).

According to previous observations by Philippe et al., it is likely that this critical flow rate demarcates the fluidized chimney regime $\left(Q>Q_{c}\right)$ and the fluidized cavity regime $\left(Q<Q_{c}\right)$. However, in this later regime, a fluidized chimney can be eventually obtained provided that one waits a sufficient time. This new regime is different from the regular one $\left(Q>Q_{c}\right)$ regarding the transient time $T_{0}$ which increases by several orders of magnitude as can be seen in Figure 3. Analyzing the time evolution of the fluidized area height $h_{f}$ obtained by some image processing, it is observed in Figure 3that, in this ultra-slow regime, the upward expansion is initially almost frozen, and consequently hardly to identify, and accelerate very progressively over about 4 to 5 decades in terms of expansion rate. This regime is also characterized by a bad reproducibility and a sharp increase of the standard deviation obtained for $T_{0}$. By comparison, the kinetics of fluidized expansion is faster and much better reproducible but also quite more regular with only a limited variation of acceleration during the transient phase as seen in Figure 3. Finally, as a consequence arising from the identification of this new regime, the real existence of a steady fluidized cavity regime is questionable, such situations being potentially

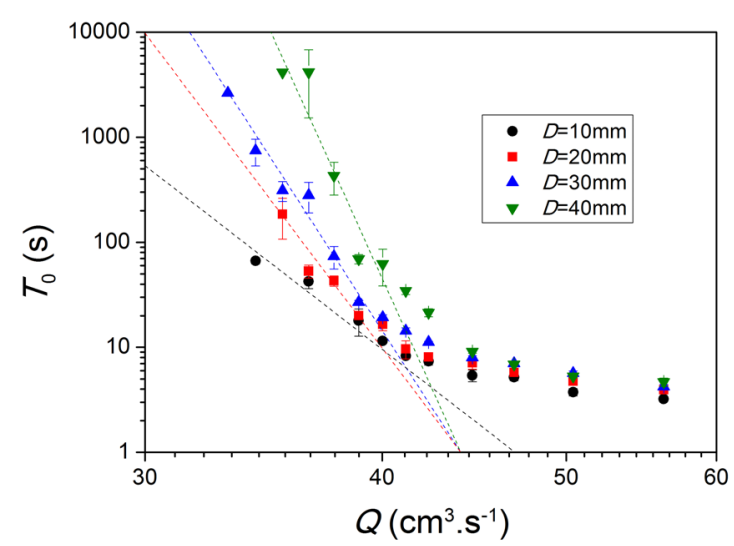

Figure 4. Log-log representation of the transient duration $T_{0}$ versus injection flow rate $Q$ for $d=3 \mathrm{~mm}, H_{0}=125 \mathrm{~mm}$, and for $D=10 \mathrm{~mm}, D=20 \mathrm{~mm}, D=30 \mathrm{~mm}$, and $D=40 \mathrm{~mm}$, respectively. The dashed lines are only guides for the eyes suggesting a linear relation between $\ln T_{0}$ and $\ln Q$.

a nearly frozen intermediate step of a complete fluidized chimney expansion. However, it has been observed that a chimney can come back to a cavity state when the flow rate is decreased [4] suggesting that a stationary fluidized cavity truly exists. In practice, the time range needed to decide on this issue is unfortunately out of any realistic experiments capability.

\subsection{Influence of the control parameters}

Regarding the ultra-slow regime, the transient duration seems to increases almost exponentially with the flow rate as suggested by the roughly linear trend in the semilogarithmic representation in Figure 4: $\ln T_{0} \propto \ln Q$. Several experimental series carried out with the four different injection diameter $D$ have clearly underlined a strong influence of $D$ on $T_{0}$. Indeed, there is a sharp increase of the slope between $\ln T_{0}$ and $\ln Q$ when the injection diameter gets wider, giving rise, for a same flow rate, to a significantly longer transient phase for a larger injection width. This effect is most probably due to a smaller velocity at injection when the diameter is enlarged that considerably reduces the expansion rate at the beginning of the fluidization. The end of the expansion is likely to be quite less dependent on the injection diameter $D$ since, far enough from injection, the size of the chimney gets almost the same whatever the value of $D$ [4].

A more systematic study was conducted in the regular regime, relying in particular on the divergence law in Eq. (1). It can first be established a weak influence of the injection diameter $D$ on the apparent critical flow-rate $Q_{c}$ : the later indeed slightly increases with $D$. Here again, this is likely due to a more moderate flow at injection for a larger injection width, which may slow down the beginning of the expansion kinetics. The transient duration is consequently increased, giving rise a slightly anticipated apparent divergence. A noticeable impact of $D$ was also 


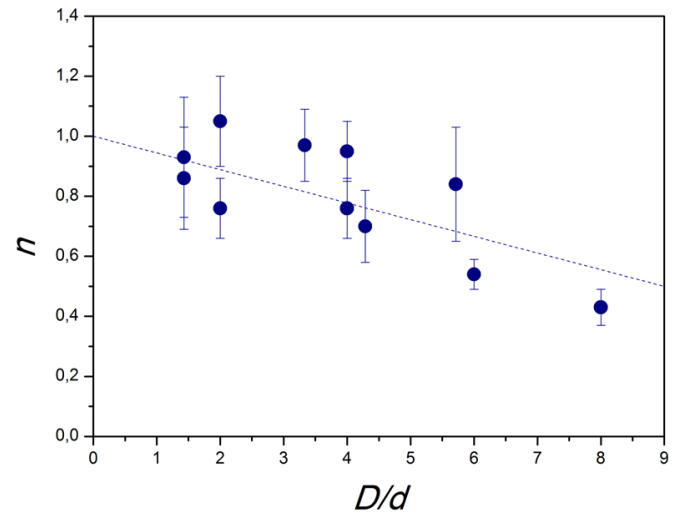

Figure 5. Plot of the exponent $n$ of the divergence law in Eq.(1) versus the ratio $\frac{D}{d}$ of the injection width to the bead diameter. The dashed blue line stands for the linear relation given in Eq.(2).

found regarding the exponent $n$ of the divergence law. This exponent actually decreases monotonously with $D$, varying for instance from $n \approx \frac{2}{3}$ for $D=10 \mathrm{~mm}$ to $n \approx \frac{1}{3}$ for $D=40 \mathrm{~mm}$ in the case of an initial sample of height $H_{0}=125 \mathrm{~mm}$ with bead diameter $d=3 \mathrm{~mm}$. Considering now the influence of the bead diameter $d$, it was observed that larger values of $n$ are obtained for larger bead diameter. Thus, for a same sample height but with beads of diameter $d=5 \mathrm{~mm}$, the range of variation for $n$ is now from $n \approx 1$ for $D=10 \mathrm{~mm}$ to $n \approx \frac{2}{5}$ for $D=40 \mathrm{~mm}$. Practically, a convenient way to satisfactory rationalize both dependencies of $n$ with $D$ and $d$ respectively can be proposed through the following relation:

$$
n \approx 1-0.056 \frac{D}{d}
$$

As can be seen in Figure 5, a rough agreement is obtained when testing the previous equation with the whole set of data.

Finally, a quadratic relation between the apparent divergence flow-rate $Q_{c}$ and the bead diameter $d$ was naturally recovered. This is simply the consequence of the fact that fluidization onset is not controlled by the flow rate transiting through the granular bed but by the pressure gradient induced by this flow. According to Darcy law (which is almost valid in the whole set of experiments since the particle Reynolds does not exceed 3 [6]), flow rate and pressure gradient are directly related through the permeability which is indeed proportional to the square of the bead diameter (see for instance Carman-Kozeny relation in [6]). Note that a complete dimensional analysis of localized fluidization accounting notably for this effect has been proposed more in details in [7].

\section{Conclusion}

By way of summary, several important findings can be underlined. First, a new and original transient regime to fluidized chimney has been highlighted and was characterized by an expansion of the fluidized area at injection that initially grows extremely slowly, in a quasi-frozen fluidized cavity configuration, before the kinetics accelerates very progressively till a final burst comparable to the more usual transient process observed in the already identified regular regime where the transient duration is related to the injection flow rate by a divergence law. On the contrary, this new "ultra-slow" regime is badly reproducible in terms of transient duration and the whole expansion process can last up to one hour or more which could explain why it has not been identified before [4]. A second significant result concerns the influence of both the injection width $D$ and the bead diameter $d$ on the transient kinetics. The injection diameter $D$ was proved to have a huge influence on the duration of the transient phase in the "ultra-slow" regime which increases dramatically with $D$, probably because, for a same flow-rate $Q$, the local velocity at injection is significantly smaller and much less efficient in fluidizing the particles. In the regular expansion regime, based on a systematic use of the divergence law of duration time versus flow-rate, it can be reported that the apparent critical flow-rate $Q_{c}$ scales with $d^{2}$, as an obvious impact of permeability, and increases slightly with injection port width $D$, and that the exponent $n$ decreases almost linearly with the ratio $D / d$.

\section{Acknowledgements}

Fundings from NSF OISE 0968313 and from the region PACA (Provence-Alpes-Côte d'Azur, France) are gratefully acknowledged.

\section{References}

[1] M.J. Rhodes. Introduction to particle technology. New York: Wiley (1998).

[2] S. Bonelli (Ed.), Erosion in Geomechanics Applied to Dams and Levees. Wiley-ISTE (2013)

[3] F. Zoueshtiagh, A. merlen, Phys. Rev. E 75(5) 053613 (2007)

[4] P. Philippe, M. Badiane, Phys. Rev. E. 87(4) 042206 (2013)

[5] J.A. Dijksman, F. Rietz, K.A. Lorincz, M. van Hecke, W. Losert. Rev. Sci. Instrum. 83011301 (2013).

[6] J. Bear. Dynamics of Fluids in Porous Media. Dover (1988).

[7] S. Mena, L.-H. Luu, P. Cuéllar, P. Philippe, J. S. Curtis. (Submitted). 